\title{
The Graphite Calorimeter as a Standard of Absorbed Dose for Cobalt-60 Gamma Radiation
}

\author{
John S. Pruitt,* Steve R. Domen,* and Robert Loevinger*
}

National Bureau of Standards, Washington, DC 20234

May 6, 1981

\begin{abstract}
Absorbed dose to water in a cobalt-60 gamma-ray beam has been determined using a thick-walled graphite ionization chamber. The chamber was calibrated in a graphite phantom against a graphite calorimeter, and the graphite calibration factor was converted to a water calibration factor using published energy absorption coefficient ratios and a measured replacement factor. Comparisons between the graphite and water measurements were made at pairs of points that were scaled in position according to the ratio of electron densities, so that the photon spectra were the same for the two points in a given pair. Measurements performed in graphite over a wide range of phantom depths, field sizes, and source distances, showed that the calibration factor varies slowly with the phantom depth and field size, and probably has a negligible dependence on source distance. By comparison with the thick-walled chamber in a cobalt-60 gamma-ray beam, a secondary ionization chamber can be calibrated in terms of absorbed dose to water with an estimated uncertainty of about \pm 1 percent.
\end{abstract}

Key words: absorbed dose standard; cobalt-60 gamma rays; electron density; ionization chamber; graphite calorimeter; scaling theorem; water phantom.

\section{Introduction}

It has long been recognized that calorimetry offers, in principle, the most direct method of determining absorbed dose. Practical calorimeter materials (e.g., graphite) were not, however, the media of interest (e.g., water). In 1969, it was proposed [1] ${ }^{1}$ that a thin-walled ionization chamber be calibrated in the calorimeter medium and this calibration be transferred to a water phantom with ionization-chamber measurements in water, using stopping-power ratios and perturbation factors. The basic limitations of this method are (1) the need for a chamber wall strong enough for practical use and thin enough to have a negligible influence on the chamber current, (2) the relatively large uncertainties in the stopping-power ratios ( \pm 2 percent), and (3) the difficulty in evaluating the perturbation factors.

Recent work has proceeded along three lines. One approach uses a small thimble-shaped graphite calorimeter for direct measurements of absorbed dose in a water phantom [2]. This technique avoids the need for a transfer ionization chamber between two different media, and consequently avoids the use of stopping-power ratios, but the perturbation factors remain.

\footnotetext{
- Center for Radiation Research, National Measurement Laboratory.
}

Another approach is exemplified by a proposal from the National Physical Laboratory [3] that the transfer instrument used between the calorimeter medium and water be a thick-walled ionization chamber. This technique avoids the need to know stopping-power ratios if it is known that photon fluence spectral distributions are similar in the two media. This proposal suggests the use of a tungsten ion chamber that would be thick-walled for photon energies up to about $10 \mathrm{MeV}$.

The third approach uses a calorimeter of water, which avoids all three of the limitations mentioned above. A water calorimeter for determination of absorbed dose has been constructed and tested at the National Bureau of Standards [4], but, at the time of this writing, results are still preliminary.

The present work was performed to establish an NBS standard for absorbed dose to water in a cobalt 60 gammaray beam, traceable to a graphite calorimeter. The work was done with a graphite ion chamber [5], shown in cross section in figure 1 , which is thick-walled for photons with energies up to about $\mathrm{l} \mathrm{MeV}$. Since the work was performed with colbalt 60 radiation, the transfer technique used is similar to that proposed by NPL, with no need for stopping-power ratios and only a small error (less than 0.15 percent) for contributions to the chamber current from electrons originating outside the chamber.

\footnotetext{
${ }^{2}$ Figures in brackets indicate literature references at the end of this paper.
} 


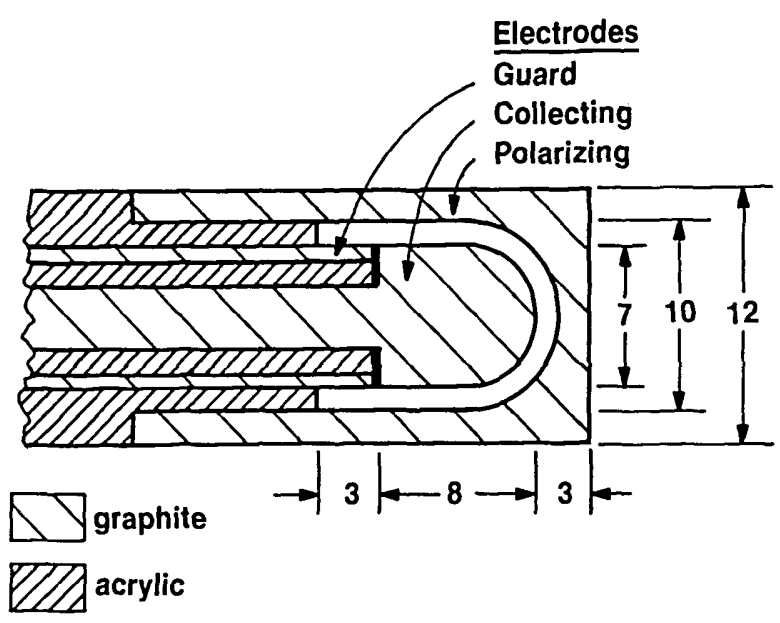

Mylar washer

FicURE 1. Schematic cross section of type PLl graphite ionization chamber. Dimensions are in millimeters. From [5].

The absorbed-dose calibration factor for an ionization chamber in an absorbing medium is the quotient of the absorbed-dose rate at the position of the reference point of the chamber in the undisturbed medium (i.e., with the chamber replaced by the medium), and the current from the chamber when it is irradiated in the medium. In the work reported here, an absorbed-dose calibration factor in graphite was obtained using a graphite calorimeter; from the graphite factor, an absorbed-dose calibration factor in water was calculated; and from that calibration factor, absorbed-dose rate to water was obtained by multiplication by the chamber current. Use was made of a photon-fluence scaling theorem [6] which assures that the spectra of primary and scattered photons have the same energy and angle dis. tributions in the graphite and the water phantoms. The perturbation factor is evaluated by an extrapolated replacement technique in which the effect of replacing graphite by water is measured experimentally.

\section{The Calibrations in Graphite}

Calibration measurements were made in two graphite phantoms, one containing a graphite calorimeter and the other the graphite ionization chamber. These phantoms were irradiated separately by a beam of cobalt 60 gamma rays in the geometry shown schematically in figure 2 . For a given set of calibrations, the source-detector distance $z$ was held constant while the field size $f$ (the field was square) and the phantom depth $x$ were varied. Both phantoms consisted of cylindrical blocks, $30 \mathrm{~cm}$ in diameter and about $17 \mathrm{~cm}$ thick. In each case the center of the detector was about $1 \mathrm{~cm}$ below the front surface of the block and the phantom depth

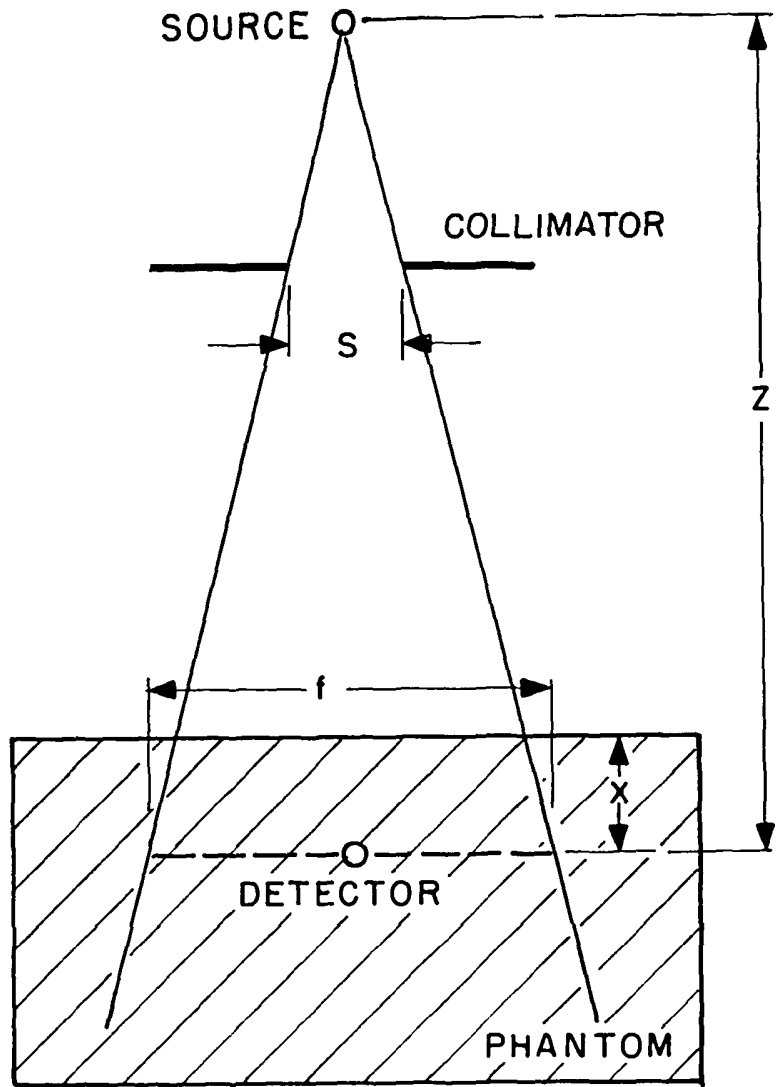

Ficure 2. Schematic diagram showing experimental parameters used in the text.

was varied by the addition of extra graphite plates, with densities between 1.65 and $1.72 \mathrm{~g} / \mathrm{cm}^{3}$.

Most of the calibration measurements were made with a $10-\mathrm{kCi}$ cobalt -60 source in our laboratory. In this case, the two phantoms were moved alternately into the beam and the same extra plates added in the same order to each in turn. The earliest calibrations were made with a $0.5-\mathrm{kCi}$ source in our laboratory, and an intermediate set of calibrations was made with a $5-\mathrm{kCi}$ source at the National Institutes of Health. All these earlier calorimeter measurements preceded the ion-chamber measurements by weeks or months, and were corrected for decay using a half-life of 5.271 years [7].

Field sizes and source-detector distances were varied only with the $10-\mathrm{kCi}$ source, where field sizes were reproduced accurately by setting the collimator jaws with metal jigs. The relation between collimator size and field size for the $10-\mathrm{kCi}$ source was determined from ionization-chamber profile measurements in air, using only one collimator size and one source distance, and assuming direct proportionality between field size $f$ and the product of collimator size $s$ and source distance $z$. 
Dose rates were measured with the NBS portable graphite calorimeter [8], and ion-chamber currents were measured with a commercial high-gain electrometer and a digital voltmeter. The mode of calorimeter operation differed slightly from that of the ionization chamber because of the need to determine beam-off drift rates before and after each calorimeter run. Calorimeter irradiation times were determined by the beam shutter system. These times required a small correction to agree with ionizationchamber integration times, which were determined electronically without turning off the beam. The correction was never larger than 0.1 percent for the NBS sources and 0.5 percent for the NIH source.

Table 1 is a list of field sizes for all the combinations of source distance and collimator size for which calibrations were made. The chamber calibration factors are listed in table 2 . In both tables, the collimator sizes listed for the $0.5-\mathrm{kCi}$ source and for the $5-\mathrm{kCi}$ source are synthetic numbers. They represent what the collimator size would have been for the $10-\mathrm{kCi}$ source to produce the field sizes measured for these two sources.

TABLE 1. Field sizes in graphite $f(\mathrm{~mm})$.

\begin{tabular}{|c|c|c|c|c|c|c|c|}
\hline \multirow{4}{*}{$\begin{array}{c}\text { Source } \\
\text { Distance } \\
z \\
(\mathrm{~m})\end{array}$} & \multicolumn{7}{|c|}{ Nominal source activity $(\mathrm{kCi})$} \\
\hline & 10 & 10 & 10 & 5 & 10 & 0.5 & 10 \\
\hline & \multicolumn{7}{|c|}{ Collimator size $s(\mathrm{~mm})$} \\
\hline & 24.0 & 28.0 & 33.4 & 34.1 & 40.5 & 43.0 & 50.8 \\
\hline 0.654 & & 52 & 62 & & 75 & & 95 \\
\hline 0.800 & & & 76 & & & & \\
\hline 0.900 & & & 86 & & & & \\
\hline 1.000 & & 80 & 95 & 97 & 115 & 123 & 145 \\
\hline 1.100 & & & 105 & & & & \\
\hline 1.200 & & & 114 & & & & \\
\hline 1.250 & 86 & 100 & 119 & & 144 & & 181 \\
\hline
\end{tabular}

For the $10-\mathrm{kCi}$ source, field sizes were calculated from $f=2.85 \mathrm{~s} z$. For the other sources, field sizes were measured.

TABLE 2. Calibration factor of chamber PLI-11 (mGy/nC at $22^{\circ} \mathrm{C}$ and 1 standard atmosphere).

\begin{tabular}{|c|c|c|c|c|c|c|c|c|}
\hline \multirow{4}{*}{$\begin{array}{c}\text { Source } \\
\text { Distance } \\
\quad z \\
(\mathrm{~m})\end{array}$} & \multirow{4}{*}{$\begin{array}{c}\text { Graphite } \\
\text { Depth } \\
e^{x} \\
\left(\mathrm{~g} / \mathrm{cm}^{2}\right)\end{array}$} & \multicolumn{7}{|c|}{ Nominal source activity $(\mathrm{kCi})$} \\
\hline & & 10 & 10 & 10 & 5 & 10 & 0.5 & 10 \\
\hline & & \multicolumn{7}{|c|}{ Collimator size $s(\mathrm{~mm})$} \\
\hline & & 24.0 & 28.0 & 33.4 & 34.1 & 40.5 & 43.0 & 50.8 \\
\hline \multirow[t]{7}{*}{0.654} & 1.65 & & 101.4 & 101.5 & & 101.6 & & 101.7 \\
\hline & 3.18 & & 101.0 & 101.2 & & 101.3 & & 101.5 \\
\hline & 5.84 & & 100.4 & 100.7 & & & & \\
\hline & 5.87 & & & & & 101.0 & & 101.2 \\
\hline & 8.37 & & 100.4 & 100.6 & & 100.7 & & 100.9 \\
\hline & 11.42 & & 100.1 & & & & & \\
\hline & 11.59 & & & 100.5 & & 100.5 & & 100.7 \\
\hline 0.800 & 5.08 & & & 101.1 & & & & \\
\hline 0.900 & $"$ & & & 101.2 & & & & \\
\hline \multirow[t]{12}{*}{1.000} & 0.86 & & & & & & 101.7 & \\
\hline & 1.65 & & 101.6 & 101.8 & 101.7 & 101.8 & & 102,0 \\
\hline & 3.18 & & 101.3 & 101.4 & 101.4 & 101.8 & & 101.6 \\
\hline & 4.06 & & & & & 101.7 & 102.4 & \\
\hline & 5.08 & & & 101.2 & & 101.4 & 101.7 & \\
\hline & 5.84 & & & 101.1 & & & & \\
\hline & 5.87 & & 101.0 & 101.2 & 101.1 & 101.4 & & 101.4 \\
\hline & 6.08 & & & & & & 101.9 & \\
\hline & 8.16 & & & & 101.0 & & & \\
\hline & 8.37 & & 100.7 & 101.0 & & 101.4 & & 101.4 \\
\hline & 9.05 & & & & & & 101.5 & \\
\hline & 11.59 & & 100.6 & 101.1 & 100.7 & 101.2 & & 101.3 \\
\hline 1.100 & 5.08 & & & 101.3 & & & & \\
\hline 1.200 & $"$ & & & 101.4 & & & & \\
\hline \multirow[t]{6}{*}{1.250} & 1.65 & 101.6 & 102.1 & 101.8 & & 101.8 & & 101.8 \\
\hline & 3.18 & 101.5 & 101.5 & 101.4 & & 101.7 & & 101.6 \\
\hline & 5.84 & 101.0 & 101.0 & 101.1 & & 101.2 & & 101.4 \\
\hline & 6.38 & & 101.3 & & & & & \\
\hline & 8.37 & 101.0 & 100.9 & 101.0 & & 101.4 & & 101.5 \\
\hline & 11.42 & 100.7 & 100.8 & 101.0 & & 101.1 & & 101.4 \\
\hline
\end{tabular}


The calibration factors of table 2 at each source distance $z$ have been fitted to an equation of the type:

$$
\begin{gathered}
N_{\text {graph }}=N_{g r a p h}^{r o f}\left[1+k_{x}\left(1-e^{-\gamma_{x}\left(\rho x-\rho x_{r}\right)}\right)+\right. \\
\left.k_{f}\left(1-e^{-\gamma_{f}\left(f-f_{r}\right)}\right)\right]
\end{gathered}
$$

where $\varrho$ is the mass density of the phantom, $N_{\text {graph }}$ is the calibration factor in absorbed dose per unit charge at depth $\boldsymbol{x}$ and field size $f$, and $N_{z r a p h}^{\text {rof }}$ is the calibration factor at reference depth $x_{r}$ and reference field size $f_{\text {r. }}$ Exponential representation was chosen arbitrarily on the assumption that the calibration factor approaches a limiting value for either large fields or large depths.

The values of the parameters and the coefficients obtained from the curve-fitting procedure are listed in table 3 , along with the coefficient of variation $V$ of each fit. As can be seen from the latter, eq (l) is a satisfactory description of the dependence of $N_{\text {graph }}$ on phantom depth and field size for the NBS $10-\mathrm{kCi}$ and the NIH sources. The larger coefficient of variation for the $0.5-\mathrm{kCi}$ NBS source is caused by larger scatter in the relatively small calorimeter signals, rather than failure of eq (1). The three values at $z=1 \mathrm{~m}$ have been combined to form a weighted mean of $N_{g r a p h}^{\text {ref }}(1 \mathrm{~m})$ $=101.27 \mathrm{mGy} / \mathrm{nC}$ at $22{ }^{\circ} \mathrm{C}$ and one standard atmosphere, with a coefficient of variation of 0.08 percent.

Figure 3 shows the dependence of the calibration factor on distance, depth, and field size, as predicted by eq (1), varying one parameter while holding the others at their reference values $\left(z_{r}=1 \mathrm{~m}, x_{r}=5 \mathrm{~g} / \mathrm{cm}^{2}, f_{r}=100 \mathrm{~mm}\right)$. The ordinate is the change from the reference calibration factor $N_{g r a p h}^{r e f}$. The three points in figure 3 are the predictions of eq (1) at $0.654,1.000$, and $1.250 \mathrm{~m}$, showing that variation with

TABLE 3. Curve-fitting parameters and coefficients, and coefficients of variation for eq (1), at $22^{\circ} \mathrm{C}$ and one standard atmosphere. $\gamma_{x}=0.25 \mathrm{~cm}^{2} / \mathrm{g}$ and

\begin{tabular}{|c|c|c|c|c|c|c|c|}
\hline $\begin{array}{l}\text { Source location and } \\
\text { nominal activity }\end{array}$ & $\begin{array}{c}\text { Distance } \\
z \\
(\mathrm{~m}) \\
\end{array}$ & $\begin{array}{c}N_{\text {refeph }}^{\text {ref }} \\
(\mathrm{mG} / \mathrm{nC})\end{array}$ & $\begin{array}{c}e^{x_{r}} \\
\left(\mathrm{~g} / \mathrm{cm}^{2}\right)\end{array}$ & $\begin{array}{c}f_{r} \\
(\mathrm{~mm})\end{array}$ & $k_{x}$ & $k_{f}$ & $\begin{array}{c}V \\
(\%)\end{array}$ \\
\hline NBS, $10 \mathrm{kCi}$ & 0.654 & 100.8 & 5.56 & 65.4 & -0.00443 & 0.00569 & 0.1 \\
\hline . & 1.000 & 101.3 & 5.00 & 100.0 & -0.00363 & 0.00384 & 0.1 \\
\hline $\mathrm{NBS}, 0.5 \mathrm{kCi}$ & * & 101.6 & 5.00 & 100.0 & -0.00363 & 0.00384 & 0.5 \\
\hline $\mathrm{NIH}, 5 \mathrm{kCi}$ & " & 101.2 & 5.00 & 100.0 & -0.00363 & 0.00384 & 0.1 \\
\hline $\mathrm{NBS}, 10 \mathrm{kCi}$ & 1.250 & 101.2 & 5.00 & 100.0 & -0.00356 & 0.00269 & 0.2 \\
\hline
\end{tabular}
$\gamma_{f}=0.025 \mathrm{~mm}^{-1}$.

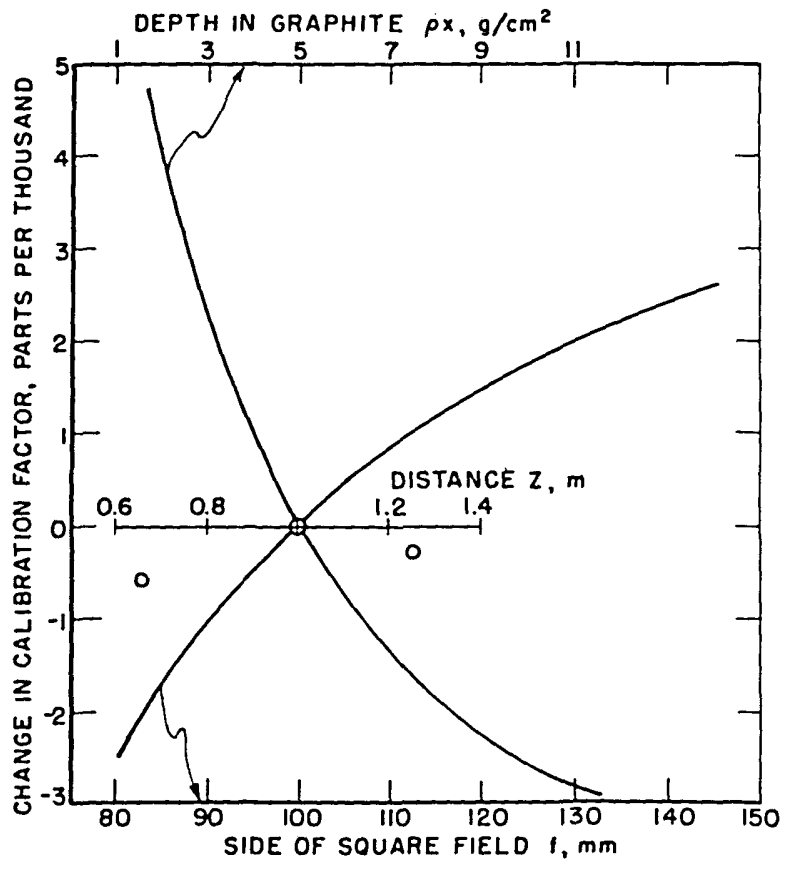

Ficure 3. Variation of PL1-11 graphite calibration factor with phantom depth, field size, and source distance, as predicted by eq (1).

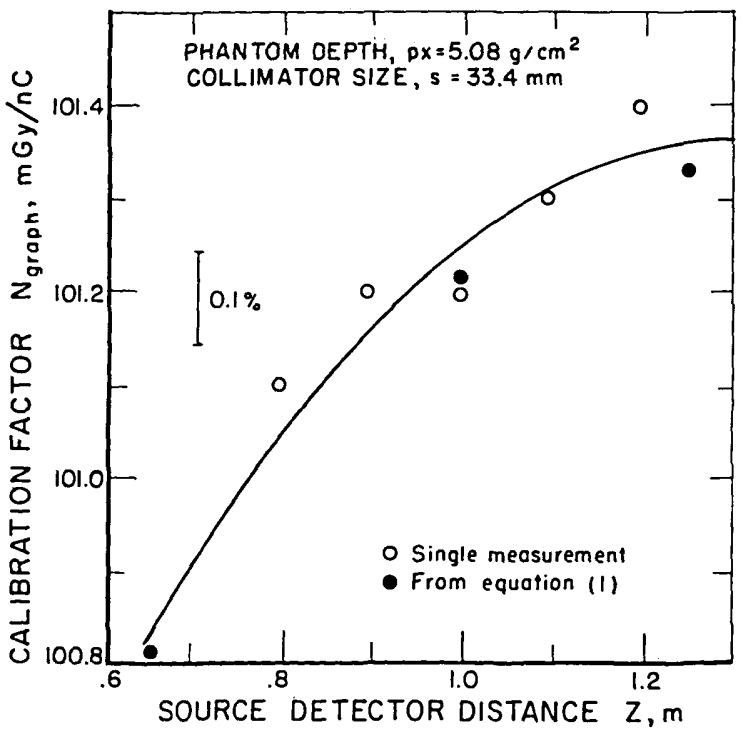

FIGURE 4. Comparison of the graphite calibration factor as predicted by eq (1) with measured calibration factors at phantom depth $5.08 \mathrm{~g} / \mathrm{cm}^{2}$ and collimator size $33.4 \mathrm{~mm} \times 33.4 \mathrm{~mm}$. The line is a parabolic least squares fit to all eight points. 
distance alone is small, and is within the uncertainty of measurement. Plots similar to figure 3 at 0.654 and $1.250 \mathrm{~m}$ look quite similar.

Finally, the calibration factors at $\varrho x=5.08 \mathrm{~g} / \mathrm{cm}^{2}$ and $s$ $=33.4 \mathrm{~mm}$ are compared with the prediction of eq (l) at $0.654,1.000$, and $1.250 \mathrm{~m}$ in figure 4 . The agreement is within 0.1 percent.

\section{Calibration Transfer Theory}

Consider an ionization chamber in a absorbing and scattering medium irradiated by a cobalt 60 gamma-ray beam. The chamber is assumed to be thick-walled, i.e., all electrons reaching the cavity arise in the chamber walls, and is further assumed to be made of a single, but unspecified, wall material. An expression for the absorbed dose to the undisturbed medium at the position of the reference point of the chamber, i.e., in the absence of the chamber, can be obtained from eqs (23) and (24) of reference [9]. Omitting terms that relate only to the properties of the wall and the cavity gas, we obtain

$$
D_{\text {med }} \propto J_{g a s}(\beta)_{\text {wall }}^{\text {med }}\left(\bar{\mu}_{\text {en }} / \varrho\right)_{\text {wall chamb }}^{\text {med }}(\Psi)_{\text {wall }}^{\text {med }}
$$

In eq (2) $J_{\text {gas }}$ is the charge per unit mass of cavity gas corresponding to $D_{\text {med }} . \beta$ is the quotient of absorbed dose and the collision part of kerma, and $(\beta)_{\text {wall }}^{\text {med }}$ is the ratio $\beta_{\text {med }} / \beta_{\text {wall }}$. $\left(\vec{\mu}_{e n} / \varrho\right)_{\text {wall }}^{\text {med }}$ is the ratio of the mean mass energy-absorption coefficient of the medium to that of the wall material. $\Psi$ is the photon energy fluence at the chamber center, and ${ }_{\text {chamb }}(\Psi)_{\text {wall }}^{\text {med }}$ is a factor that corrects for the replacement of the medium by wall material in the volume of the entire chamber, wall plus cavity.

The calibration factor for the chamber in the medium is proportional to $D_{\text {med }} / J_{\text {gas }}$. Assume that the chamber has been calibrated in a cobalt- 60 gamma-ray beam in graphite, and is user in water at a point where the photon spectral energy fluence is the same as in the graphite. Then expressions for $N_{\text {graph }}$ and $N_{\text {water }}$ can be obtained from eq (2), and the ratio of these expressions gives

$$
N_{\text {water }}=N_{\text {graph }}(\beta)_{g r a p h}^{\text {water }}\left(\bar{\mu}_{\text {en }} / \varrho\right)_{\text {graph chamb }}^{\text {water }}(\Psi)_{g r a p h}^{\text {water }}
$$

Equation (3) provides the required relationship between the calibration factor $N_{\text {graph }}$ determined in graphite using the graphite calorimeter, and the desired calibration factor $N_{\text {water }}$ that applies in undisturbed water. The energyabsorbtion coefficients are averaged over the spectral energy fluence in the undisturbed medium, at the point of measurement. The replacement factor corrects for the replacement of water by graphite in the volume of the entire chamber. More explicitly, the replacement factor is

$$
{ }_{\text {chamb }}(\Psi)_{\text {graph }}^{\text {water }}=\Psi_{\text {water }} / \Psi_{\text {graph }}
$$

where $\Psi_{\text {water }}$ is the photon energy fluence at the position of the chamber center in undisturbed water, i.e., in water in the absence of the chamber; and $\Psi_{\text {graph }}$ is the photon energy fluence in water at the same position inside a piece of graphite that has the same outer dimensions as the ionization chamber.

The derivation of eq (3) required that the spectral energy fluence be the same in water and in graphite, at the measurement points. It has been shown [6] that this can be achieved by scaling all dimensions in the inverse ratio of the electron densities in order to define corresponding points in the two media, and then comparing measurements only at pairs of corresponding points.

Equation (3) is independent of the wall material of the ionization chamber. This is the basis of the proposal [3] to use a high-density material for the ionization chamber, so as either to reduce the size of the chamber (which in turn causes the replacement factor to be closer to unity), or to increase the energy range within which the chamber can be considered thick-walled, or both. The designation "graph" in eqs (3) and (4) refers to the graphite of the phantom, not to the graphite of which the chamber was constructed.

\section{Experimental Realization of Calibration Transfer}

Values of $\beta$ for cobalt- 60 gamma radiation calculated for polystyrene, carbon, and air, assuming secondary electron equilibrium, do not differ by more than 0.1 percent $[9$, app. B]. Therefore it is assumed that the ratio $(\beta)_{\text {graph }}^{\text {water }}$ in eq (3) can be taken as unity, with an uncertainty of about 0.1 percent.

The mean mass energy-absorption coefficient at a point in a scattering medium is a function of the photon spectral energy fluence at that point. The spectral energy distribution varies with both field size and depth in the medium, as then does the mean mass energy-absorption coefficient. The ratio of coefficients at corresponding scaled points in two media varies with field size and depth much more slowly than do the coefficients themselves. This ratio was evaluated as a function of depth using the cobalt 60 spectra in graphite of Seltzer, Hubbell, and Berger (an example of which is shown in figure 26 of reference [10]), and the graphite and the water mass energy-absorption coefficients of Hubbell [11]. The variation with field size was deduced using the cobalt- 60 spectra in water of Bruce and Johns [12], normalized to graphite for one field size. Figure 5 shows the result: variation of the ratio is almost negligible over the range of depths and field sizes of interest here. 


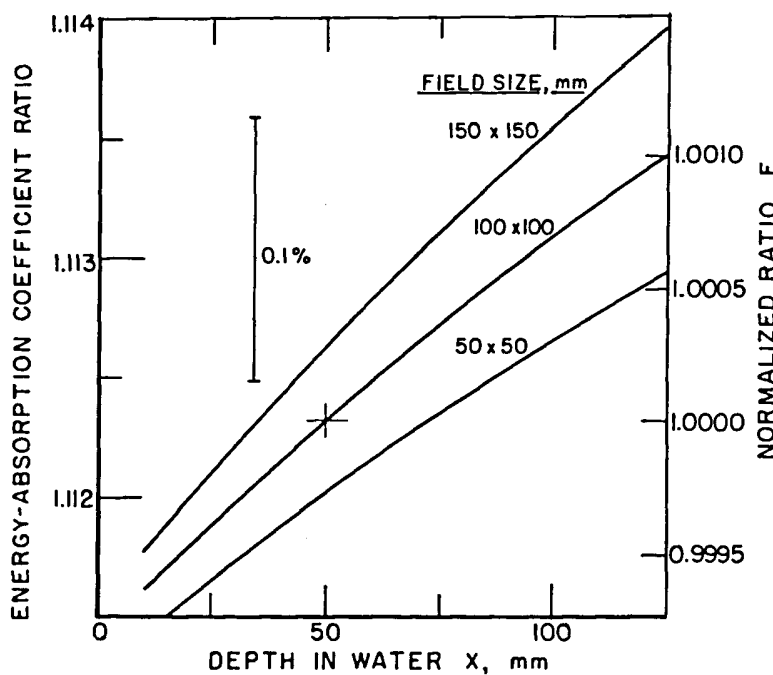

FIGURE 5. Ratio of the mean mass energy-absorption coefficients of water and of graphite, as a function of field size and depth in a water phantom.

The replacement factor in eq (3) was evaluated experimentally, using the graphite ionization chamber in a water phantom. The outer diameter of the chamber was increased by addition of cylindrical graphite sleeves, replacing water by graphite outside the chamber. It was found that the chamber current decreased at the rate of 0.068 percent per millimeter increase in diameter. ${ }^{2}$ Assuming that the same rate of change of current can be extrapolated to the chamber center, the replacement factor is then calculated from the $12.5-\mathrm{mm}$ chamber diameter as 1.0085 . From figure 5 , the energy-absorption coefficient ratio for a square field $100 \mathrm{~mm}$ on a side, at a depth of $50 \mathrm{~mm}$ in water, is 1.1123 . Then eq (3) can be written in the form

$$
N_{\text {water }}(z, x, f)=1.122 F(x, f) N_{\text {graph }}\left(z^{\prime}, x^{\prime}, f^{\prime}\right)
$$

for the NBS graphite chamber designated PL1. In eq (5), $F(x, f)$ is the energy-absorption coefficient ratio at a depth $x$ and a field size $f$ in water, divided by the ratio at a depth of $50 \mathrm{~mm}$ and a field size of $100 \mathrm{~mm} . F(x, f)$ is shown on the right-hand scale of ordinates in figure 5 , and is assumed to be independent of source distance $z . N_{\text {graph }}\left(z^{\prime}, \mathrm{x}^{\prime}, \mathrm{f}^{\prime}\right)$ is to be evaluated from eq (l) for the values $z^{\prime}, x^{\prime}, f^{\prime}$ that correspond to $z, x, f$, according to the scaling rule used here.

The scaling rule [6] requires that all distances be scaled inversely as the number of electrons per unit volume. The mass densities of water and the graphite used in the phantom were taken to be $1.00 \mathrm{~g} / \mathrm{cm}^{3}$ and $1.70 \mathrm{~g} / \mathrm{cm}^{3}$, respectively. The number of electrons per unit volume are then 0.555 $N_{A}$ and $0.849 N_{A}$, where $N_{A}$ is Avogadro's number, and the scaling factor is 1.530 . Then $z^{\prime}=z / 1.530$, and similarly for $x^{\prime}$ and $f^{\prime}$.

This number contains corrections for the difference between the average graphite phantom density, $1.70 \mathrm{~g} / \mathrm{cm}^{3}$, and the $\mathrm{graphite}$ sleeve density $1.77 \mathrm{~g} / \mathrm{cm}^{3}$.
The reference conditions at NBS for an absorbed-dose calibration in water are source distance $z=1 \mathrm{~m}$, depth in water $x=50 \mathrm{~mm}$, and field size $f=100 \mathrm{~mm}$. The corresponding scaled distances in graphite are in the first line of table 3. An expression for the calibration factor as a function of depth in water and field size is then obtained from eqs (1) and (5) in the form

$$
\begin{aligned}
N_{\text {water }}(1, x, f)=N_{\text {water }}^{\text {ref }} F(x, f)[1 & +k_{x}\left(1-e^{-\xi_{x}(x-50)}\right) \\
& \left.+k_{f}\left(1-e^{-\xi_{f}(f-100)}\right)\right]
\end{aligned}
$$

where $N_{\text {water }}^{\text {ref }}=N_{\text {water }}(1,50,100)=113.1 \mathrm{mGy} / \mathrm{nC}$ at $22^{\circ} \mathrm{C}$ and 1 standard atmosphere. The coefficients in eq (6) are $k_{x}$ $=-0.00443, k_{f}=0.00569, \xi_{x}=0.028 \mathrm{~mm}^{-1}$, and $\xi_{f}=$ $0.016 \mathrm{~mm}^{-1}$.

\section{Calibration Accuracy}

The absorbed-dose calibration of chamber PL1-11 can be transferred to another (secondary) ionization chamber by substitution in a water phantom in the cobalt- 60 gamma-ray beam. The component uncertainties that enter into the absorbed-dose rate to water and into the calibration of the secondary chamber are given in table 4 . The "statistical" uncertainties $s_{i}$ are values of the coefficient of variation (the standard deviation in percent), estimated from repeated measurements. These include the uncertainties that are conventionally identified as random. The "other" uncertainties $w_{i}$ are estimated values of the upper bound (expressed in percent) of all possible errors that have been identified but cannot be assigned a coefficient of variation. These include the uncertainties that are conventionally identified as systematic, but may include some that are conventionally identified as random.

The dominant systematic uncertainty in table 4 is that for the energy-absorption coefficient ratios. The uncertainty quoted is based on Hubbell's estimate [11] of an uncertainty of \pm 0.3 percent for monoenergetic photons in the cobalt -60 energy range, increased to \pm 0.5 percent because of the presence of low-energy photons, for which the ratio of the coefficients is much less accurately known.

For some purposes, it is convenient to combine the statistical and the other uncertainties into an estimated overall uncertainty. In the absence of a rigorous theory to guide formation of an overall uncertainty, several approaches are presented here, and the results compared. According to conventional statistical theory, the combined variance of a distribution is obtained by adding the variances of the component distributions. Then the component coefficients of variation are combined in quadrature to obtain an overall coefficient of variation:

$$
s=\sqrt{\sum s_{i}^{2}}
$$


TABLE 4. Uncertainty analysis.

\begin{tabular}{ccc}
\hline & \multicolumn{2}{c}{ Estimated } \\
Component uncertainty & \multicolumn{3}{c}{ uncertainly (\%) } \\
\cline { 2 - 3 } & statistical & other \\
& $s_{i}$ & $w_{i}$ \\
\hline
\end{tabular}

1. Calorimeter [13]

Impurities during calibration

Impurities during irradiation

Measurement of calibration power

0.1

0.02

$0.04 \quad 0.03$

2. PL1-11 chamber in graphite

Calibration factor

0.15

0.1

Position uncertainty, chamber vs calorimeter

Lack of full wall buildup [6]

3. Dose-rate conversion, graphite to water

Absorption coefficient ratio [11]

$\beta$ ratio

Replacement factor

0.2

4. Calibration of secondary chamber in water

$\begin{array}{lll}\text { Measurement of current } & 0.1 & 0.1 \\ \text { Chamber shape, possible airgaps, etc. } & & 0.1 \\ \text { Position uncertainty, secondary vs } & & 0.03\end{array}$

PLl chamber

0.03

\begin{tabular}{ll} 
Absorbed-dose rate to water (1 to 3) & \\
Linear combination, eq (8) & 1.8 \\
Quadratic combination, eq (10) & $0.8(1.1)$ \\
& \\
Calibration of secondary chamber (1 \\
$\quad$ to 4) \\
Linear combination, eq (8) \\
Quadratic combination, eq (10) & 2.1 \\
\hline
\end{tabular}

Suppose now that a factor $k_{1}$ is chosen so that the confidence level of the individual products $k_{1} s_{i}$ is approximately the same as that believed to be associated with the individual estimated $w_{i}$. Then the component uncertainties can be combined linearly to give an overall uncertainty in the form

$$
U=k_{1} s+\Sigma w_{i}
$$

An expression for the overall uncertainty is sometimes obtained by combination of the component uncertainties in quadrature, based on a direct analogy with eq (8), in the form

$$
U=\sqrt{\left(k_{1} s\right)^{2}+\sum w_{i}^{2}}=k_{1} \sqrt{s^{2}+\sum\left(w_{i} / k_{1}\right)^{2}}
$$

Equation (9) is essentially a combination in quadrature of confidence limits, which has no theoretical justification.

It has recently been suggested [14] that each $w_{i}$ be adjusted by a suitable factor $k_{2}$ so that it becomes plausible to treat the terms $\left(k_{2} w_{i}\right)^{2}$ as if they were variances. Then the overall uncertainty takes the form

$$
U=k_{1} \sqrt{s^{2}+\Sigma\left(k_{2} w_{i}\right)^{2}}
$$

The value of $k_{2}$ depends on the form assumed for the population underlying the $w_{i}$, which is usually not known. If it is assumed that the distribution of possible errors is normal, $k_{2}=k_{1}^{-1}$ and eq (10) becomes identical to eq (9).

The "other" uncertainties $w_{1}$ have been estimated here as realistic upper bounds of the possible errors, so it is reasonable to assume that the $w_{1}$ correspond very roughly to 99 percent confidence limits. Therefore we choose $k_{2}=1 / 3$ and $k_{1}=3$. Then from eqs (8), and (9) or (10), we obtain the overall uncertainties shown in table 4 . It is sometimes argued that a single large uncertainty should be combined linearly with the remaining uncertainties combined in quadrature. When this is done with the uncertainty in the absorption-coefficient ratio, we obtain the values shown in parentheses in table 4 .

Combination of uncertainties in quadrature is chosen over linear combination, since it is considered to be very unlikely that all errors would occur in the same sense. Since the component uncertainties are at best rough estimates and may have been underestimated, and noting the considerable variations in values of the overall uncertainty obtained using different methods of calculation, secondary chamber calibrations based on this work will be stated to have an overall uncertainty of about \pm 1 percent. It does not appear to be justified to specify the overall uncertainty to two significant figures.

We are grateful to the physics staff of the Radiation Oncology Department of the National Cancer Institute of the National Institutes of Health for making their cobalt- 60 source available for these studies. We are also grateful to Ronald Collé for many helpful discussions on statements of uncertainty.

\section{References}

[1] Radiation dosimetry: $\mathbf{x}$-rays and gamma rays with maximum photon energies between 0.6 and $50 \mathrm{MeV}$. Int. Comm. on Rad. Units and Meas. (Wash., D.C.) Report 14; 1969 September. 30 p.

[2] Sundara Rao, I.S.; Naik, S. B. Graphite calorimeter in water phantom and calibration of ionization chambers in dose to water for ${ }^{60} \mathrm{Co}$ gamma radiation. Med. Phys. 7(3): 196-201; 1980 May-June.

[3] Burns, J. E. Conversion of calibrations from absorbed dose to carbon into absorbed dose to water for high energy $x$ radiation. Nat. Phys. Lab. (Teddington, U.K.) Report RS 50; 1980 August.

[4] Domen, Steve R. Absorbed dose water calorimeter. Med. Phys. 7(2): 157-159; 1980 March-April. 
[5] Pruitt, J. S.; Loevinger, R. Ionization chamber for absorbed-dose calibration. Proceedings of Symposium on Measurements for the Safe Use of Radiation; 1976 March 1-4; Gaithersburg, Md. Nat. Bur. Stand. (U.S.) Spec. Publ. 456: 37-39; 1976 November.

[6] Pruitt, J. S.; Loevinger, R. The photon-fluence scaling theorem for Compton-scattered radiation. Submitted to Med. Phys.

[7] A handbook of radioactivity measurements procedures. Nat. Comm. on Rad. Protec. and Meas. (Wash., D.C.) Report 58; 1978 November. $506 \mathrm{p}$.

[8] Domen, Steve R.; Lamperti, Paul J. A heat-loss compensated calorimeter: theory, design, and performance. J. Res. Nat. Bur. Stand. (U.S.) 78A(5): 595-610; 1974 September-October.

[9] Loevinger, Robert. A formalism for calculation of absorbed dose to a medium from photon and electron beams. Med. Phys. 8(1): 1-12; 1981 January-February.

[10] Seltzer, S. M.; Hubbell, J. H.; Berger, M. J. Some theoretical aspects of electron and photon dosimetry. Proceedings of the International
Atomic Energy Agency International Symposium on National and International Standardization of Radiation Dosimetry; 1977 December 5-9; Atlanta, Ga. Int. Atomic En. Agency (Vienna) 1978, 3-43.

[11] Hubbell, J. H. Photon mass attenuation and mass energy absorption coefficients for $\mathrm{H}, \mathrm{C}, \mathrm{N}, \mathrm{O}, \mathrm{Ar}$, and seven mixtures from $0.1 \mathrm{keV}$ to $20 \mathrm{MeV}$. Rad. Res. 70(1): 58-81; 1977 April.

[12] Bruce, W. R.; Johns, H. E. The spectra of x-rays scattered in low atomic number materials. Brit. J. Rad. (London) Supplement 9; 1960. 57 p.

[13] Domen, S. R.; Lamperti, P. J. Comparisons of calorimetric and ionometric measurements in graphite irradiated with electrons from 15 to $50 \mathrm{MeV}$. Med. Phys. 3(5): 294-301; 1976 SeptemberOctober.

[14] Collé, Ronald. Personal communication concerning a meeting of a Working Group on the Statement of Uncertainties, Bureau International des Poids et Mesures (Sèvres) 1980 October. 